\title{
Weighted-Power-Mean Mixture Model for the Gibbs Energy of Fluid Mixtures
}

\author{
Walter W. Focke* \\ Institute of Applied Materials, Department of Chemical Engineering, University of Pretoria, \\ Pretoria 0002, South Africa
}

A continuous family of excess Gibbs free energy expressions is derived based on the double-weighted power mean mixture model and concepts from cubic equations of state. This family has two types of parameters: The model form is determined by two power indices, whereas the matrix of binary coefficients characterizes pure component behavior and binary interactions. It is shown that the Porter, Margules, and Wassiljewa composition dependence are but special forms of this more general expression. In particular, the Wassiljewa Gibbs free energy form turns out to have the same composition dependence as the nonrandom two-liquid (NRTL) equation.

\section{Introduction}

The Gibbs free energy of an $n$-component liquid mixture can be partitioned as follows:

$$
G=G^{\mathrm{E}}+\sum_{i=1}^{n} x_{i} G_{i}+R T \sum_{i=1}^{n} x_{i} \ln x_{i}
$$

Here, $G_{i}$ is the Gibbs free energy of pure component $i, G^{\mathrm{E}}$ is the excess Gibbs free energy, and the $x_{i}$ is the mole fraction of component $i$ in the mixture. It is convenient to rewrite eq 1 in dimensionless form by scaling with respect to $R T$ :

$$
g \equiv \frac{G}{R T}=g^{\mathrm{E}}+\sum_{i=1}^{n} x_{i} g_{i}+\sum_{i=1}^{n} x_{i} \ln x_{i}
$$

The dimensionless excess Gibbs energy $g^{\mathrm{E}}=g^{\mathrm{E}}(T, P, \mathbf{x})$ expresses the nonideal behavior of liquid mixtures as a function of temperature, pressure, and mixture composition. At low to medium pressures, the pressure dependence is usually weak and frequently ignored. ${ }^{1} g^{\mathrm{E}}$ reflects the effect of differences between the intermolecular interactions in the pure components and in the mixture and also the structural changes that accompany mixing. ${ }^{2}$ Knowledge of its temperature, pressure, and composition dependence provides a complete description of the thermodynamic properties of the mixture. Unfortunately, thermodynamics does not provide an explicit functional form for the temperature and composition dependence of the excess Gibbs free energy. Consequently one has to turn to empirical and semirational approaches. ${ }^{1}$ Early significant $g^{\mathrm{E}}$ empirical descriptions were provided by Margules, ${ }^{3}$ van Laar, ${ }^{4}$ Porter, ${ }^{5}$ Wohl, ${ }^{6}$ and Redlich and Kister. ${ }^{7}$ The most important semitheoretical equations are based on the theoretical concept of local compositions. They include the Wilson, ${ }^{8}$ NRTL, ${ }^{9}$ and UNIQUAC ${ }^{10}$ equations. However, Flemr ${ }^{11}$ and McDermot and Ashton ${ }^{12}$ showed that the local composition theory, as implemented in these equations, is actually inconsistent for nonrandom mixtures.

The composition dependences of the Porter, ${ }^{5}$ Margules, ${ }^{3}$ and NRTL equations differ ostensibly. Nevertheless, this communication intends to show that these classic $g^{\mathrm{E}}$ expressions are just special cases of a more general mixing rule recently developed for fluid physical properties. ${ }^{13,14}$ Toward this end, suitable expressions for the following function will be developed:

* To whom correspondence should be addressed. Fax: +27 12 4202516. E-mail: walter.focke@up.ac.za.

$$
g^{\#}=\frac{G}{R T}-\sum_{i=1}^{n} x_{i} \ln x_{i}=\frac{G^{\mathrm{E}}}{R T}+\sum_{i=1}^{n}\left(\frac{G_{i}}{R T} x_{i}\right)=g^{\mathrm{E}}+\sum_{i=1}^{n} x_{i} g_{i}
$$

It will be shown that an equation with NRTL-like composition dependence can be obtained without invoking the local compositions concept.

\section{Weighted-Power-Mean Mixture Model (WPM3) ${ }^{13}$}

A liquid is a condensed phase characterized by a degree of short-range molecular order and the absence of any long-range order. $^{2}$ Accordingly, the local liquid structure about a central reference molecule may be defined by the cell formed by the shell of nearest neighbors. ${ }^{15,16}$ The molecular size and shape as well as the strength of the intermolecular interactions determine the cell size and the relative spatial orientations of the adjacent molecules. ${ }^{2,17}$ Orientation structuring is important when the molecules possess a dipole moment. For example, liquid water is highly structured. A strong preference is shown for tetrahedral orientation of hydrogen atoms about oxygen atoms owing to directional hydrogen bonding. ${ }^{2}$

Additional complications arise when liquid mixtures are considered. ${ }^{8,18}$ Differences in the shape or size of the unlike molecules are expected to affect the molecular arrangement in the mixture. ${ }^{19,20}$ Differences in the attractive forces between like and unlike molecules are especially important. ${ }^{8}$ Competition among the various molecular species, in their interaction with the central molecule, results in local compositions that may differ from the bulk concentrations. Wilson ${ }^{8}$ used a Boltzmann-type distribution function to describe the relocation of molecules in the shell of nearest neighbors.

The fact that intermolecular interactions have an extremely short range justifies the assumption that only binary interactions need to be considered. This allows a fluid mixture to be viewed as an assembly of hypothetical fluid clusters defined by the nature of a central molecule together with its nearest neighbors. ${ }^{8,9}$ Every fluid physical property is then ultimately determined by the binary interactions between the central molecules and their cluster neighbors. Focke et al. ${ }^{13}$ postulated that the effect of these interactions, on the values that a given physical property will assume, can be captured by a matrix of binary coefficients $C=\left[c_{i j}\right]$. In their notation, the subscript $i$ specifies the nature of the central molecule of the cluster and $j$ the nature of a neighboring molecule. In a pure component $i$ fluid, all of the 
interactions are identical and their effect on the fluid physical property is described by the parameter $c_{i i}$. It immediately follows that $c_{i i}$ is equivalent to the fluid physical property of pure component $i$, that is $c_{i i}=c_{i}$.

In the above framework, a mixture rule is defined by prescriptions for (i) estimating fluid cluster properties and (ii) how to combine them to yield the overall mixture property value. A particularly flexible form is obtained when both prescriptions entail global composition-weighted power means. ${ }^{13}$ This yields the weighted-power-mean mixture model of orders $r$ and $s$, which for $r, s \neq 0$ reads:

$$
f(C, \mathrm{x})=\left[\sum_{i=1}^{n} x_{i}\left(\sum_{j=1}^{n} x_{j} c_{i j}^{s}\right)^{r / s}\right]^{1 / r}
$$

Equation 4 features two types of adjustable parameters: The $c_{i j}$ are adjustable binary coefficients while the model constants $r$ and $s$ determine the nature of the composition dependence. For preselected $(r, s)$ values, the resulting model predicts, in principle at least, multicomponent behavior from knowledge of pure component and binary mixture data. The choice $r=1$ reduces eq 4 to the neural network mixture model proposed by Focke. ${ }^{14}$

Consider the physical interpretation of the model constants $r$ and $s$. In essence, $s$ defines the averaging process for calculating cluster property values. The parameter $r$ prescribes the averaging process for determining the mixture property value in terms of the cluster values. For instance, when these indices take on the special values 1,0 , or -1 , the corresponding averaging processes correspond to composition weighted arithmetic, geometric, and harmonic means. In both cases, the weighing is done with respect to bulk compositions.

Before eq 4 can be applied as a mixing rule for the Gibbs free energy, it is necessary to find suitable interpretations for the coefficients $c_{i j}$ and to postulate a link between the functions $g^{\#}$ and $f(C, \boldsymbol{x})$. The possibility that $g^{\#}=-\ln f(C, \boldsymbol{x})$ will be considered in a separate communication but in this study it is simply assumed that:

$$
g^{\#} \equiv f(C, x)=\left[\sum_{i=1}^{n} x_{i}\left(\sum_{j=1}^{n} x_{j} c_{i j}^{s}\right)^{r / s}\right]^{r}
$$

Again, choosing $r=1$ implies that the composition dependence of the function $g^{\#}$ is defined by Focke's neural network mixture model. ${ }^{14}$

Equation 4 is homogeneous of order one in the coefficients $c_{i j}$, that is for all $\lambda$ :

$$
f\left(\left[\lambda c_{i j}\right], x\right)=\lambda f\left(\left[c_{i j}\right], x\right)=\lambda f(C, x)
$$

The homogeneity property ensures that the model is dimensionally homogeneous and guarantees that it is intrinsically wellconditioned with respect to all the adjustable binary coefficients $c_{i j}{ }^{14}$ Furthermore, the choice $\lambda=-1$ shows that it is permissible for all of the coefficients of the matrix $C$ to be negative:

$$
f\left(\left[-c_{i j}\right], x\right)=-f\left(\left[c_{i j}\right], x\right)=-f(C, x)
$$

However, except for special values for $r$ and $s$, the model is not invariant with respect to arbitrary scale translations, that is

$$
f\left(\left[c_{i j}-\alpha\right], x\right) \neq f\left(\left[c_{i j}\right], x\right)-\alpha
$$

Thus, eq 4 applies only to properties with well-defined absolute values. This clearly poses a dilemma when the Gibbs free energy is considered as it is usually defined with respect to some (arbitrary) reference state. Fortunately it is possible to find an unambiguous absolute representation, suitable for use in this formalism, from cubic equations of state (EoS).

\section{Excess Gibbs Energy Expressions from Cubic Equations of State}

Huron and $\mathrm{Vidal}^{21}$ proposed a generalized form for the van der Waals-type cubic equation of state with $\phi_{1}$ and $\phi_{2}$ as numerical constants:

$$
P=\frac{R T}{(v-b)}-\frac{a}{\left(v+\varphi_{1} b\right)\left(v+\varphi_{2} b\right)}
$$

For pure components, the attraction parameter $a=a(T)$ and the covolume $b$ can be evaluated from critical properties and vapor pressure data. For mixtures, appropriate mixing rules are required to describe the composition dependence of the parameters $a_{\text {mix }}$ and $b_{\text {mix }} \cdot{ }^{1,22,23}$ For example, the classic van der Waals rules read:

$$
a_{\text {mix }}=\sum_{i} \sum_{j} a_{i j} x_{i} x_{j} \text { and } \quad b_{\text {mix }}=\sum_{i} b_{i} x_{i}
$$

Here, the $a_{i i}$ and $b_{i}$ are pure component EoS parameter values.

Wong and Sandler ${ }^{24}$ postulated that the excess Helmholtz free energy of mixing is pressure independent. This implies that the excess Helmholtz free energy of an equation of state (EoS), evaluated at infinite pressure, provides a reasonable approximation for the excess Gibbs free energy in the liquid state:

$$
\begin{aligned}
& G^{\mathrm{E}}\left(T, x_{i}\right)=G^{\mathrm{E}}\left(T, P \approx 0, x_{i}\right) \approx A_{\mathrm{EOS}}^{\mathrm{E}}\left(T, P \rightarrow 0, x_{i}\right) \approx \\
& A_{\mathrm{EOS}}^{\mathrm{E}}\left(T, P \rightarrow \infty, x_{i}\right)
\end{aligned}
$$

The infinite pressure state corresponds to a situation where there is no free volume. Thus, as $P \rightarrow \infty, v \rightarrow b$, and $v_{i i} \rightarrow b_{i i}$, that is the molar volume of the mixture and the molar volumes of the pure components respectively equal the covolume parameters $b$ and $b_{i}{ }^{23,24}$ With these conditions, the excess Helmholtz energy at infinite pressure, that is the excess Gibbs energy, reduces to: ${ }^{21,23}$

$$
g^{\mathrm{E}} \approx \frac{A_{\infty}^{\mathrm{E}}}{R T}=-\frac{\Phi}{R T} \frac{a_{\mathrm{mix}}}{b_{\operatorname{mix}}}-\sum_{i=1}^{n}\left(-\frac{\Phi}{R T} \frac{a_{i i}}{b_{i}}\right) x_{i}
$$

$\Phi$ is a characteristic constant of the cubic equation of state used. ${ }^{21}$ For $\phi_{1}=\phi_{2}, \Phi=1 /\left(1+\phi_{1}\right)$, whereas $\Phi=\ln [(1+$ $\left.\left.\phi_{1}\right) /\left(1+\phi_{2}\right)\right] /\left(\phi_{1}-\phi_{2}\right)$ when $\phi_{1} \neq \phi_{2}$.

From eq 12, it is inferred that

$$
g^{\#}=g^{\mathrm{E}}+\sum_{i=1}^{n} x_{i} g_{i}=g^{\mathrm{E}}+\sum_{i=1}^{n}\left(-\frac{\Phi}{R T} \frac{a_{i i}}{b_{i}}\right) x_{i}=-\frac{\Phi}{R T} \frac{a_{\mathrm{mix}}}{b_{\mathrm{mix}}}
$$

Comparing eq 5 with eq 12 or with eqs 10 and 13 at a composition limit $x_{i} \rightarrow 1$ (i.e., corresponding to pure component i) reveals the following rational link between EoS parameters and the constants $c_{i i}$ of the weighted-power-mean mixture model:

$$
c_{i i}=-\frac{\Phi}{R T} \frac{a_{i i}}{b_{i}}
$$

Because $a_{i i}, b_{i}>0$, it follows that $c_{i i}<0$ when $\Phi>0$.

\section{Wassiljewa or Two-Parameter NRTL Model}

Renon and Prausnitz ${ }^{9}$ derived the nonrandom two-liquid (NRTL) model. To account for the nonrandomness of liquid mixtures, the classic NRTL model invokes Scott's two-liquid 
model $^{17}$ and Wilson's "local compositions" concept. ${ }^{8}$ The intermolecular interactions, $g_{i j}$, are construed as Gibbs free energies with the $g_{i i}$ as the Gibbs free energy of the pure substance $i{ }^{1}$ It is further assumed that $g_{i j}=g_{j i}$. The local distribution of molecules in the immediate vicinity of another molecule is then expressed in probabilistic terms by a Boltzmann-type distribution based on the differences in the interaction energies. This endows the model parameters with the following implicit temperature dependence:

$$
G_{i j}=\exp \left(-\alpha \tau_{i j}\right) \quad \text { and } \quad \tau_{i j}=\left(g_{i j}-g_{j j}\right) / R T
$$

Renon and Prausnitz ${ }^{9}$ considered $\alpha$ to be an empirical constant. In the context of Guggenheim's quasichemical theory $\alpha=1 / z$, that is it should be inversely proportional to the coordination number in the liquid state. Walas ${ }^{1}$ recommends a value of $\alpha=0.4$ for aqueous organic mixtures and $\alpha=0.3$ for nonaqueous mixtures. Marina and $\operatorname{Tassios}^{25}$ found that setting $\alpha=-1$ provided good representations of binary mixture behavior. In general, the NRTL equation represents highly nonideal multicomponent equilibrium data, for example aqueous-organic systems, quite well. ${ }^{1,26}$ For a binary mixture, the NRTL model is given by:

$$
g^{\mathrm{E}}=x_{1} x_{2}\left[\frac{\tau_{21} G_{21}}{x_{1}+G_{21} x_{2}}+\frac{\tau_{12} G_{12}}{G_{12} x_{1}+x_{2}}\right]
$$

The Wilson and UNIQUAC models form the bases for the ASOG and UNIFAC activity coefficient prediction by group contribution methods. NRTL usually gives better data fits than UNIQUAC and unlike Wilson it can predict liquid-liquid phase separation. ${ }^{1}$ A possible handicap of NRTL is that three adjustable parameters must be fixed for each pair of constituents. This could be the reason why a predictive NRTL, based on group contribution methods, was never developed. Raal and Muhlbauer $^{26}$ state that, despite the fact that the NRTL model has a reasonable basis in theory, the link is insufficient to fix the values of the parameters on the basis of molecular considerations. It will now be shown that eq 4 predicts the same composition dependence as the NRTL equation when $r=1$ and $s=-1$ :

$$
g^{\#}=\sum_{i=1}^{n} \frac{x_{i}}{\sum_{j=1}^{n} x_{j} / c_{i j}}
$$

Setting $\Lambda_{i j}=c_{i i} / c_{i j}$ in eq 17 and rewriting it in terms of $g^{\mathrm{E}}$ yields the following revised two-parameter NRTL-like expression for multicomponent mixtures:

$$
g^{\mathrm{E}}=\sum_{i=1}^{n} c_{i i} x_{i}\left[\frac{\sum_{j=1}^{n}\left(1-\Lambda_{i j}\right) x_{j}}{\sum_{k=1}^{n} \Lambda_{i k} x_{k}}\right]
$$

Because $\Lambda_{i i}=1$, eq 18 reduces to the following expression for a binary mixture:

$$
g^{\mathrm{E}}=x_{1} x_{2}\left[\frac{c_{11}\left(1-\Lambda_{12}\right)}{x_{1}+\Lambda_{12} x_{2}}+\frac{c_{22}\left(1-\Lambda_{21}\right)}{\Lambda_{21} x_{1}+x_{2}}\right]
$$

The equivalence with the NRTL composition dependence becomes clearer when eq 19 is first recast in the following alternative form

$$
g^{\mathrm{E}}=x_{1} x_{2}\left[\frac{\Lambda_{12}\left(c_{12}-c_{11}\right)}{x_{1}+\Lambda_{12} x_{2}}+\frac{\Lambda_{21}\left(c_{21}-c_{22}\right)}{\Lambda_{21} x_{1}+x_{2}}\right]
$$

Renon and Prausnitz ${ }^{9}$ used a reverse index notation for their model parameters. Thus the conventional binary mixture NRTL form, eq 16, obtains when one sets

$$
\begin{array}{r}
\tau_{12}=c_{21}-c_{22} ; \quad \tau_{21}=c_{12}-c_{11} ; \quad G_{12}=\Lambda_{21}=c_{22} / c_{12} \\
\text { and } G_{21}=\Lambda_{12}=c_{11} / c_{12}
\end{array}
$$

It can be shown that eq 18 also features the same composition dependence as the multicomponent NRTL equation. Thus, the standard expressions for activity coefficients that are available for the classic equation can also be used for the two-parameter per binary version through the links defined by eq 21 .

Note that, in the present context, the NRTL composition dependence corresponds to the prescriptions that cluster properties be evaluated as a weighted harmonic mean and the mixture property as a weighted arithmetic mean over the cluster properties. In each case, the weighing is based on bulk mole fractions, that is to say, "local compositions" did not enter the argument at all. Interestingly Wassiljewa ${ }^{27}$ first derived this NRTL-type composition dependence as a theoretical mixture model for the thermal conductivity of dilute hard-sphere gas mixtures.

Whereas the present two-parameter formulation and the original three-parameter NRTL have a specific excess Gibbs energy composition dependence in common, that is eq 16 , they are not identical. The difference lies in the way the model parameters $\tau_{i j}$ and $G_{i j}$ are linked. Renon and Prausnitz ${ }^{9}$ derived the relation given by eq 15 , whereas in the present formulation

$$
G_{i j}=\frac{c_{j j}}{\tau_{i j}+c_{j j}}
$$

In addition, the present formalism defines the $\tau_{i j}$ and the $G_{i j}$ in terms of the corresponding EoS parameters. This implies that the parameter temperature dependence can be linked to the temperature dependence of the EoS parameters. Figure 1 compares typical variations of $G_{i j}$ with changes in $\tau_{i j}$.

Preliminary testing of the data correlating capability of the Wassiljewa-NRTL formulation revealed that, while it excels for some systems, it holds no particular advantage over the conventional NRTL model. In part, this is due to the additional flexibility imparted by the additional adjustable parameter.

\section{Composition Order}

Equation 5, with the $c_{i i}$, defined by eq 14 , describes a continuum of activity coefficient models in the $(r, s)$ Cartesian

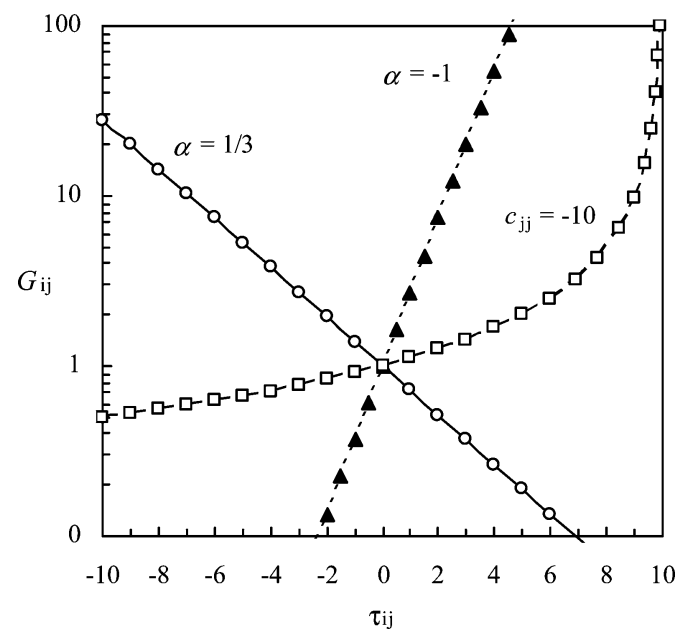

Figure 1. Illustrating the relationship between $G_{i j}$ and $\tau_{i j}$ for the classic NRTL $G_{i j}=\exp \left(-\alpha \tau_{i j}\right)$ with (i) $\alpha=1 / 3(-\bigcirc-)$, (ii) $\alpha=-1$ (-口-), and (iii) $G_{i j}=c_{j j} /\left(c_{j j}+\tau_{i j}\right)$ with $c_{j j}=10(\cdots \mathbf{\Lambda} \cdot \cdot)$. 


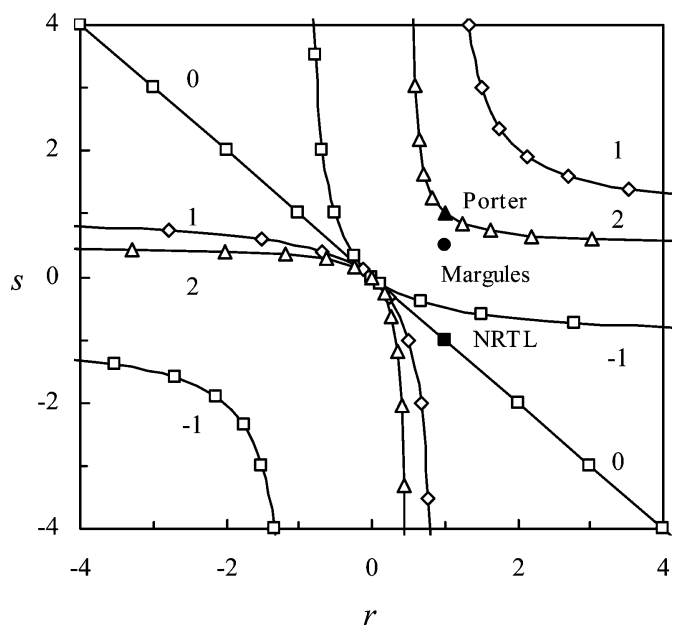

Figure 2. $(r, s)$ Cartesian plane showing the location of the classic NRTL, Margules, and Porter models. The lines represent constant compositionorder plots.

plane. ${ }^{13}$ The Porter, two-parameter Margules and WassiljewaNRTL models are special cases of eq 5 that have $r=1$ in common. This means that they are actually subsets of the neural network mixture model. ${ }^{14}$ Inspection reveals that choosing $s=$ 1 delivers the Porter ${ }^{5}$ model, whereas $s=1 / 2$ generates a twoparameter Margules equation. ${ }^{13}$ Thus, these three equations, and other yet to be explored functional forms, are simply special cases of a more general Gibbs energy model defined by eq 5 .

The nature of the composition dependence of the various models is succinctly quantified by its order. Inspection reveals that the Gibbs function $g^{\#}$ is of order

$$
n=\frac{1}{r}+\frac{1}{s}
$$

in composition, that is mole fractions. Figure 2 shows contour plots of constant composition order $n$ in the $(r, s)$ Cartesian plane. The coordinates of the Porter, two-parameter Margules, and Wassiljewa-NRTL models are also shown. The Porter model has a quadratic composition dependence $(n=2)$, whereas the Margules is third order in composition $(n=3)$. The origin in this plot $(s=r=0)$ is an attractor as contours of all orders have this point in common. It corresponds to a double-weighted geometric mean mixture model of infinite order in composition. Taking logarithms, it can be shown that the composition dependence $\ln f(C, \boldsymbol{x})$ follows the quadratic Scheffé form: ${ }^{28,29}$

$$
\ln f(C, x)=\sum_{i=1}^{n} x_{i}\left(\sum_{j=1}^{n} x_{j} \ln c_{i j}\right)
$$

Hajra $^{30}$ previously proposed excess Gibbs free energy models containing exponential functions of composition.

\section{Conclusions}

The derivation of the weighted-power-mean mixture model (WPM3) is based on the following concepts: (i) multicomponent mixtures can be viewed as a hypothetical collection of fluid clusters, and (ii) global composition-weighted power means define the prescriptions needed for estimating fluid cluster properties and combining them to yield an overall mixture property. Whereas the WPM3 predicts multicomponent properties from knowledge of pure component and binary mixture data, it only applies to absolute physical properties. This posed a problem for free energies as they are usually measured relative to some reference state. A main finding of this communication is that a suitable absolute format for the Gibbs free energy can be constructed by considering limiting forms of a cubic equation of state. The end result is eq 5 with the pure component parameters defined by eq 14. This model defines a continuous family of Gibbs free energy expressions featuring two types of parameters: The model form is determined by two power indices, whereas the matrix of binary coefficients characterizes pure component behavior and binary interactions. It was further shown that the composition dependences shown by the classic Porter, Margules, and NRTL activity coefficient models are just special forms of this more general expression. The classic models differ primarily with respect to the order of the composition dependence, which is zero, two, and three for the NRTL, Porter, and Margules models respectively. Interestingly, a model that is of infinite order in composition was identified.

\section{Acknowledgment}

Financial support for this research from the THRIP program of the Department of Trade and Industry and the National Research Foundation of South Africa as well as Xyris Technology is gratefully acknowledged.

\section{Literature Cited}

(1) Walas, S. M. Phase Equilibrium in Chemical Engineering; Butterworth: Boston, 1985.

(2) Maitland G. C.; Rigby, M.; Smith, E. B.; Wakeham, W. A. Intermolecular Forces; Clarendon Press: Oxford, 1987.

(3) Margules, M. Über die Zusammensetzung der Gesättigten Dampfe von Mischungen. Sitzb. d. mathem. naturw. Cl. 1895, 104, 1243.

(4) Van Laar, J. J. Über Dampfspannungen von Binären Gemischen. Z. Phys. Chem. 1910, 72, 723.

(5) Porter, A. W. On the Vapour-Pressures of Mixtures. Trans. Faraday Soc. 1921, 16, 336.

(6) Wohl, K. Thermodynamic Evaluation of Binary and Ternary Liquid Systems. Trans. Am. Inst. Chem. Eng. 1946, 42, 215.

(7) Redlich, O.; Kister, A. T. Algebraic Representation of Thermodynamic Properties and the Classification of Solutions. Ind. Eng. Chem. 1948, 40,345 .

(8) Wilson, G. M. Vapor-Liquid Equilibrium. XI. A New Expression for the Excess Free Energy of Mixing. J. Am. Chem. Soc. 1964, 86, 127.

(9) Renon, H.; Prausnitz, J. M. Local Compositions in Thermodynamic Excess Functions for Liquid Mixtures. AIChE J. 1968, 14, 135.

(10) Abrams, D. S.; Prausnitz, J. M. Statistical Thermodynamics of Liquid Mixtures: A New Expression for the Excess Gibbs Energy of Partly or Completely Miscible Systems. AIChE J. 1975, 21, 116.

(11) Flemr, V. A Note on the Excess Gibbs Energy Equations Based on Local Composition Concept. Collect. Czech. Chem. Commun. 1978, 41, 3347.

(12) McDermot, C.; Ashton, N. Note on the Definition of Local Composition. Fluid Phase Equilib. 1977, 1, 33.

(13) Focke, W. W.; Sandrock, C.; Kok, S. Weighted-Power-Mean Mixture Model: Empirical Mixing Laws for Liquid Viscosity. Ind. Eng. Chem. Res. 2007, 46, 4660 .

(14) Focke, W. W. Neural Network Averaging. Neural Comput. 2005, 18,1 .

(15) Lennard-Jones, J. E.; Devonshire, A. F. Critical Phenomena in Gases. I. Proc. R. Soc. London, Ser. A 1937, 163, 53.

(16) Barker, J. A. The Cell Theory of Liquids. Proc. R. Soc. London, Ser. A. 1955, 230, 390.

(17) Scott, R. L. Corresponding States Treatment of Nonelectrolyte Solutions. J. Chem. Phys. 1956, 25, 193.

(18) Prausnitz, J. M. Molecular Thermodynamics of Fluid-Phase Equilibria; Prentice-Hall: Englewood Cliffs, NJ, 1969.

(19) Huggins, M. L. Solutions of Long Chain Compounds. J. Chem. Phys. 1941, 9, 440 .

(20) Flory, P. J. Thermodynamics of High Polymer Solutions. J. Chem. Phys. 1942, 10, 51.

(21) Huron, M.-J.; Vidal, J. New Mixing Rules in Simple Equations of State for Representing Vapour-Liquid Equilibria of Strongly Non-ideal Mixtures. Fluid Phase Equilib. 1979, 3, 255. 
(22) Hicks, C. P.; Young, C. L. The Gas-Liquid Critical Properties of Binary Mixtures. Chem. ReV. 1975, 75, 119.

(23) Vidal, J. Mixing Rules and Excess Properties in Cubic Equations of State. Chem. Eng. Sci. 1977, 33, 787.

(24) Wong, D. S. H.; Sandler, S. I. A Theoretically Correct Mixing Rule for Cubic Equations of State. AIChE J. 1992, 38, 671.

(25) Marina, J. M.; Tassios, D. P. Effective Local Compositions in Phase Equilibrium Correlations. Ind. Eng. Chem. Process. Des. DeV. 1973, 12, 67.

(26) Raal, J. D.; Mu"hlbauer, A. L. Phase Equilibrium: Measurement and Computation; Taylor \& Francis: WA, 1998.

(27) Wassiljewa, A. Wa rmeleitung in Gasgemischen. Phys. Z. 1904,
(28) Scheffe', H. Experiments with Mixtures. J. R. Stat. Soc. B 1958, 20,344 .

(29) Draper, N. R.; Pukelsheim, F. Mixture Models Based on Homogeneous Polynomials. J. Stat. Plan. Inf. 1998, 71, 303.

(30) Hajra, J. P. A New Formalism for Representation of Binary Thermodynamic Data. Mater. Trans. B 1980, 11B, 215. 5,737 . 